\title{
Vacuolated Neutrophil Count
}

National Cancer Institute

\section{Source}

National Cancer Institute. Vacuolated Neutrophil Count. NCI Thesaurus. Code C74628.

The determination of the number of neutrophils containing small vacuoles present in a sample. 